\title{
Distributed feedback InGaN/GaN laser diodes
}

Thomas J. Slight, Scott Watson, Amit Yadav, Szymon Grzanka, Szymon Stanczyk, et al.

Thomas J. Slight, Scott Watson, Amit Yadav, Szymon Grzanka, Szymon Stanczyk, Kevin E. Docherty, Edik Rafailov, Piotr Perlin, Steve Najda, Mike Leszczyński, Anthony E. Kelly, "Distributed feedback InGaN/GaN laser diodes," Proc. SPIE 10532, Gallium Nitride Materials and Devices XIII, 1053219 (23 February 2018); doi: 10.1117/12.2285632 


\title{
Distributed feedback InGaN/GaN laser diodes
}

\author{
Thomas J Slight*a, Scott Watson ${ }^{\mathrm{b}}$, Amit Yadav ${ }^{\mathrm{c}}$, Szymon Grzanka ${ }^{\mathrm{d}}$, Szymon Stanczyk ${ }^{\mathrm{d}}$, Kevin E \\ Docherty $^{\mathrm{e}}$, Edik Rafailov ${ }^{\mathrm{c}}$, Piotr Perlin ${ }^{\mathrm{d}}$, Steve Najda ${ }^{\mathrm{d}}$, Mike Leszczyński $^{\mathrm{d}}$, Anthony E Kelly ${ }^{\mathrm{b}}$ \\ ${ }^{\mathrm{a}}$ Compound Semiconductor Technologies Global Ltd, Hamilton, UK, G72 0BN; ${ }^{\mathrm{b}}$ School of \\ Engineering, University of Glasgow, Glasgow, UK, G12 8LT; ${ }^{\mathrm{C}}$ Aston University, Birmingham, UK, \\ B4 7ET ; 'Topgan, Sokołowska, Warsaw, Poland ; ${ }^{\mathrm{e} K e l v i n ~ N a n o t e c h n o l o g y ~ L t d, ~ O a k f i e l d ~ A v e n u e, ~}$ \\ Glasgow, UK, G12 8LT.
}

\begin{abstract}
We have realised InGaN/GaN distributed feedback laser diodes emitting at a single wavelength in the $42 \mathrm{X} \mathrm{nm}$ wavelength range. Laser diodes based on Gallium Nitride $(\mathrm{GaN})$ are useful devices in a wide range of applications including atomic spectroscopy, data storage and optical communications. To fully exploit some of these application areas there is a need for a GaN laser diode with high spectral purity, e.g. in atomic clocks, where a narrow line width blue laser source can be used to target the atomic cooling transition. Previously, GaN DFB lasers have been realised using buried or surface gratings. Buried gratings require complex overgrowth steps which can introduce epi-defects. Surface gratings designs, can compromise the quality of the p-type contact due to dry etch damage and are prone to increased optical losses in the grating regions. In our approach the grating is etched into the sidewall of the ridge. Advantages include a simpler fabrication route and design freedom over the grating coupling strength.Our intended application for these devices is cooling of the $\mathrm{Sr}+$ ion and for this objective the laser characteristics of SMSR, linewidth, and power are critical. We investigate how these characteristics are affected by adjusting laser design parameters such as grating coupling coefficient and cavity length.
\end{abstract}

Keywords: Semiconductor lasers, Distributed feedback laser diodes, InGaN, Sidewall grating, Slotted laser, Notched grating, Lateral grating

\section{INTRODUCTION}

Gallium Nitride $(\mathrm{GaN})$ laser diodes have found a wide range of applications ranging from optical communications [1] to atomic spectroscopy [2]. To fully exploit many of these application areas there is a requirement for a GaN laser diode with high spectral purity and wavelength selectivity. For example in atomic clocks, where a narrow line width blue laser source can be used to target the atomic cooling transition [3], and in fluorescence spectroscopy for medical diagnostics where one can accurately target the emission wavelength [4].

There are several approaches that can be taken to fabricate a GaN DFB laser. Buried gratings, where the grating is formed on an epi layer close to the active region and then overgrown with cladding and contact layers, has been shown in [5]. Single wavelength operation was demonstrated although buried gratings require complex overgrowth steps which have the potential to introduce epi-defects. Surface gratings designs [6], all though simpler to fabricate, can compromise the quality of the p-type contact due to dry etch damage and are also prone to increased optical losses in the electrically un-pumped grating regions. Using shallow etched lateral grating designs, some of these issues are resolved [7], however deeply etched lateral gratings, [8], benefit from a much simpler fabrication route and have the potential for larger coupling coefficients. The authors have previously reported third and higher order sidewall gratings in the InGaN/GaN material system [9], with single wavelength emission. In this paper we summarize previous results and current efforts to improve laser performance.

*tslight@compoundsemi.co.uk

Gallium Nitride Materials and Devices XIII, edited by Jen-Inn Chyi, Hiroshi Fujioka,

Hadis Morkoç, Proc. of SPIE Vol. 10532, 1053219 - @ 2018 SPIE

CCC code: $0277-786 \mathrm{X} / 18 / \$ 18 \cdot$ doi: $10.1117 / 12.2285632$ 


\section{DESIGN}

\subsection{Third order gratings}

We have chosen to use $3^{\text {rd }}$ order gratings with $\left(\sim 120 \mathrm{~nm}\right.$ minimum feature size) as $1^{\text {st }}$ order gratings ( $\sim 40 \mathrm{~nm}$ minimum feature size) would have been too challenging to fabricate. For grating design, and to better understand the influence of grating dimensions on device performance we carried out some simple modeling. The effective modal index ( $\left.\mathrm{n}_{\text {eff }}\right)$ of our GaN laser structure was calculated using the beam propagation method. For lateral grating waveguide widths of $1.5 \mu \mathrm{m}$ and $2.5 \mu \mathrm{m}$ (W1 \& W2 in Figure 2) and an etch depth of $\sim 500 \mathrm{~nm}$ we achieve a $\Delta \mathrm{n}$ of 0.0015 . From this value of $\Delta \mathrm{n}$ and using couple mode theory we can estimate a value of coupling coefficient of $22 \mathrm{~cm}^{-1}$. For a high single mode yield, the product of $\kappa$ and cavity length should be around 1.5 which leads to an optimal cavity length in the region of $700 \mu \mathrm{m}$.

\subsection{High order gratings}

Conventional implementations of high order DFB gratings consist of slots etched into the top of the laser waveguide ridge [10]. However this approach is not suited to GaN laser diodes where the p-type GaN contact layer ideally should be continuous along the ridge to minimise contact resistance and reduce optical losses in the p-type GaN. In our design the index perturbations are formed regularly spaced notches in the ridge sidewall with the contact metallization running the entire length of the ridge.

The approach to grating design was as follows: the grating bandwidth is a function of the reflectivity of the individual notch pair, the lower the reflectivity the narrower the bandwidth but the larger the number of pairs required to maintain the required total reflectivity. For a GaN laser the effective modal index is low $(\sim 2.4)$ so the FP modes are closely spaced at $\sim 0.05 \mathrm{~nm}$ and the grating bandwidth should be of a similar value to achieve single wavelength operation. We used the TMM (transmission matrix method) along with effective modal indices found using a 2D mode solver to calculate the grating bandwidth. This technique is approximate but gives us an estimate of the required grating length. Our design used 125 notch pairs along the ridge which we estimate to have a bandwidth $0.08 \mathrm{~nm}$.

\subsection{Narrow linewidth}

One of the main aims of this work is develop narrow line width $(<1 \mathrm{MHz})$ blue laser operation suitable for implementation in quantum sensors based on ultracold atoms. To this end we are developing a blue laser linewidth measurement system, more details of which are given in section 5 . We have also examined several design approaches with the aim of decreasing laser linewidth. For the $3^{\text {rd }}$ order chips we have developed long cavity chip designs with reduced coupling coefficient, strategies demonstrated to reduce linewidth in GaInAsP/InP laser diodes [11]. High order DFB designs have been demonstrated to have intrinsically narrower linewidths than conventional DFB laser diodes [12] and so could be particularly suited to spectroscopic applications. For both types of grating we have developed designs with various cavity lengths and coupling strengths with the aim of optimizing power, efficiency and SMSR.

\section{FABRICATION}

Device fabrication was carried out on commercially available GaN laser grade material which consisted of three InGaN quantum wells with GaN barriers. The fabrication for both third order and high order gratings follows the same general process. Grating and ridge patterns were defined in ZEP 520 resist using electron beam lithography (EBL). The EBL tool used was a Vistec VB6 UHR having a write resolution of $1.0 \mathrm{~nm}$, enabling the accurate targeting of the emission wavelength. Reactive ion etching was used to transfer the pattern into a $100 \mathrm{~nm}$ thick $\mathrm{SiO}_{2}$ hard mask. Inductively coupled plasma (ICP) etching on an STS Multiplex tool was then used to form the grating and ridge. $\mathrm{A} \mathrm{Cl}_{2} / \mathrm{N}_{2}$ based ICP etch process with $300 \mathrm{~W}$ platen and $600 \mathrm{~W}$ coil power produced the vertical and smooth etch profile required for good grating performance. Fig 1 shows electron micrographs of the $3^{\text {rd }}$ and $39^{\text {th }}$ order gratings. Electrical contacting was achieved using $\mathrm{Pd} / \mathrm{Au}$ and $\mathrm{SiO}_{2}$ as an insulator for contact definition on the $\mathrm{Mg}$ doped $\mathrm{p}-\mathrm{GaN}$ cap layer. Finally, the samples were thinned by mechanical polishing and the devices were cleaved into laser cavities of various lengths with both front and back facets left uncoated. 

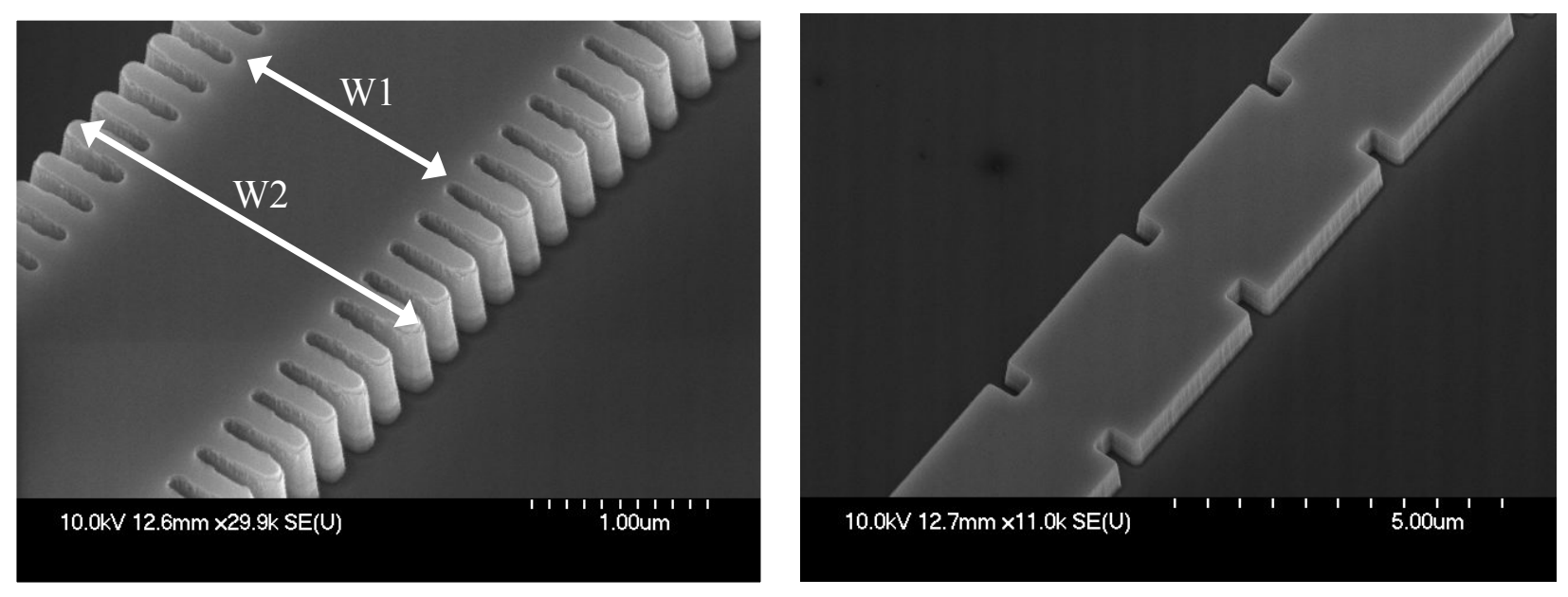

Fig.1. a) SEM image of the as etched $3^{\text {rd }}$ order grating.

b) SEM image of the as etched $39^{\text {th }}$ order grating.

\section{CHARACTERISATION}

In this section we present recent results obtained from $39^{\text {th }}$ order devices in the $42 \mathrm{X}$ nm range [13]. Previous results from $3^{\text {rd }}$ order grating chips can be found in [9].

The as cleaved lasers were characterized under pulsed drive conditions. For spectral measurements we used a Horiba iHR550 with spectral resolution of $0.025 \mathrm{~nm}$. Fig 2 shows optical power and voltage as a function of pulsed drive current for a device with grating pitch of $3548 \mathrm{~nm}$ and 125 slot pairs. Peak power is $15 \mathrm{~mW}$ measured at a drive current of 500 $\mathrm{mA}$. Notched lasers and Fabry-Perot lasers processed from the same wafer both demonstrated similarly high threshold currents which was attributed to the quality of the epi material. The slope efficiency of the FP lasers was higher $(0.48$ $\mathrm{mA} / \mathrm{mW})$ than the notched lasers $(0.13 \mathrm{~mA} / \mathrm{mW})$ which was thought to be due to scattering losses from the grating.

From fig 3a we can see that the device is lasing in a single FP mode up to drive currents of $500 \mathrm{~mA}$ before becoming multimode at higher drive currents (fig 3b). This is due to FP modes shifted from the Bragg wavelength reaching threshold as wavelength dependent losses [10] are overcome. Introducing more slot pairs with weaker index contrast would decrease the bandwidth of the grating and potentially improve single mode performance at higher drive currents.

\section{LINEWIDTH MEASUREMENT}

We are investigating two approaches for measuring the linewidth of these devices. The first makes use of a near-infrared source and a frequency doubler to convert down to blue wavelengths. A Titanium Sapphire laser with ultra-narrow linewidth $(<50 \mathrm{kHz})$ is used as a reference laser. The light from this laser is frequency doubled using a non-linear crystal to convert the wavelength to one of which is very close to that of the device under test. By tuning the device based on its current and temperature, the lasers can beat together, resulting in a beat frequency visible on the electrical spectrum analyzer. As the linewidth of the reference laser is very low, it can be assumed that what is measured is the linewidth of the laser under test. An experimental setup for this is shown below in fig 4 . 


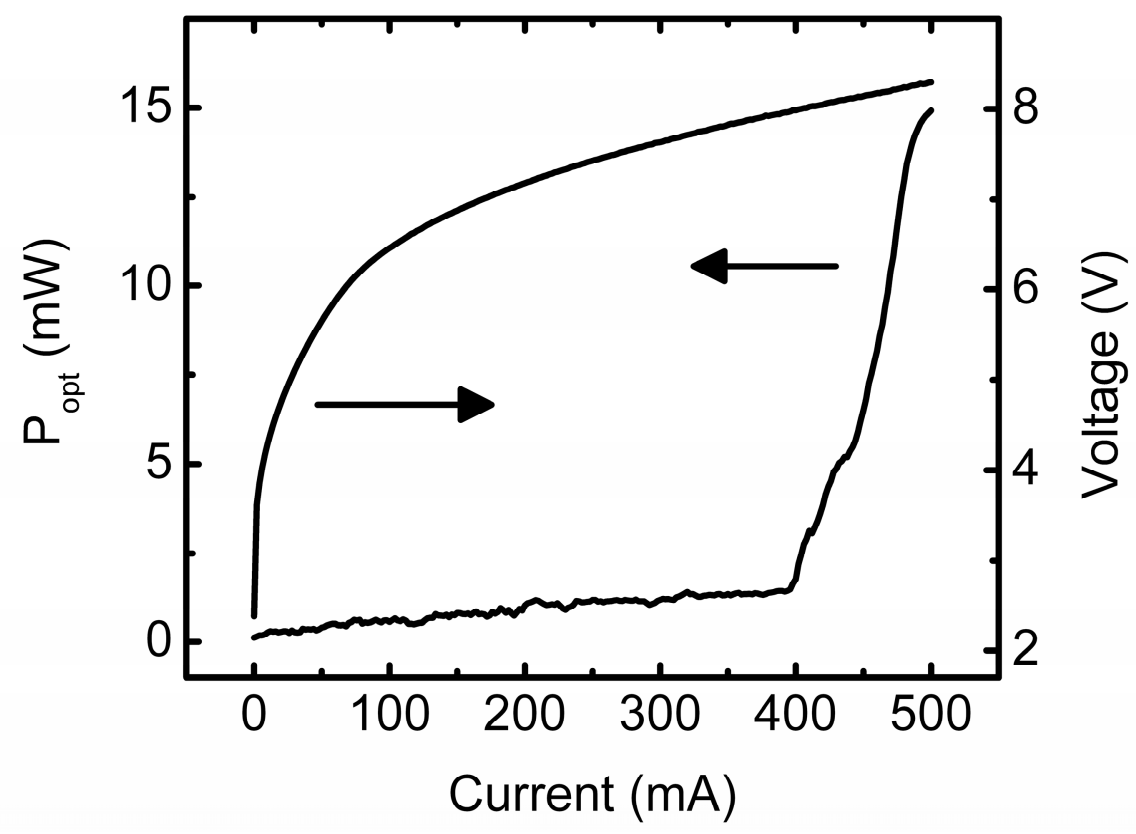

Fig .2. Optical power and voltage as a function of drive current.

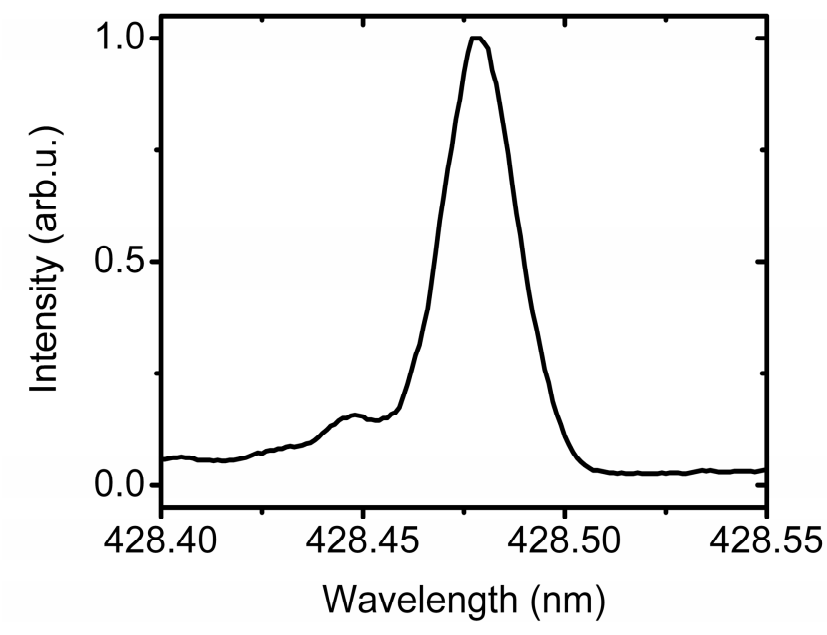

Fig.3.a) Emission spectrum at drive current of $500 \mathrm{~mA}$.

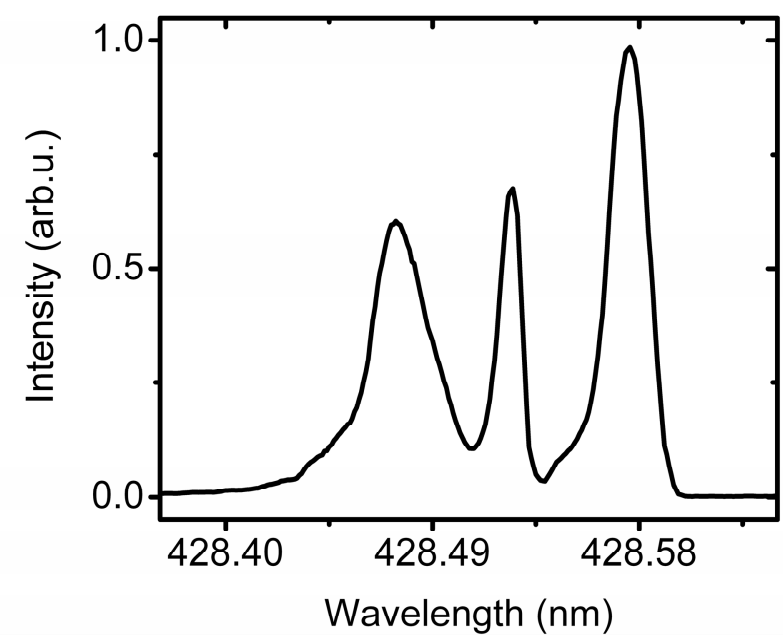

b) Emission spectrum at drive current of $600 \mathrm{~mA}$. 


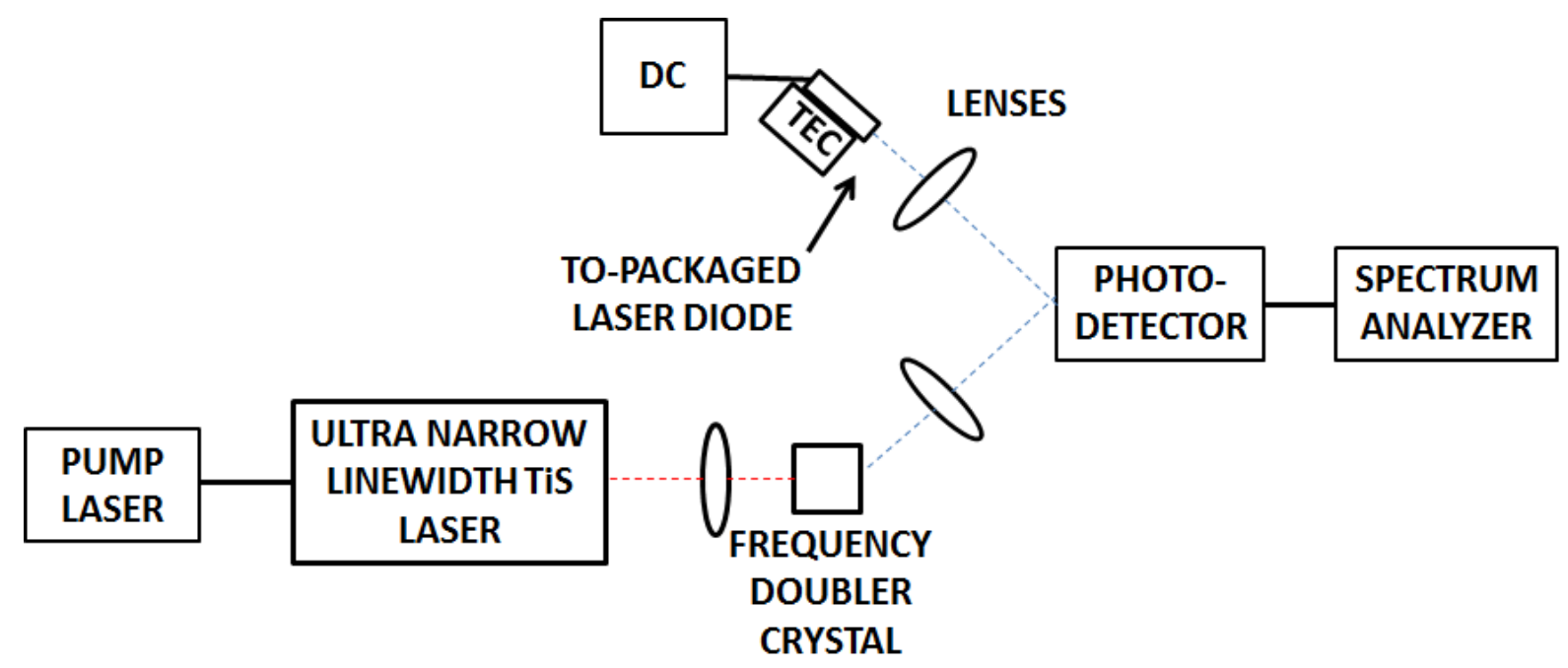

Fig.4. Frequency doubling technique to measure the linewidth of a blue laser.

These setups are bulky, complex and expensive. However, it is possible to simplify this by using two laser devices which are very similar in performance to beat together as shown in fig 5 . If the wavelengths are very close together, then a beat frequency will be detected. This is currently limited by the bandwidth of the photodetector, meaning the tuning of the current and temperature accurately is very important.

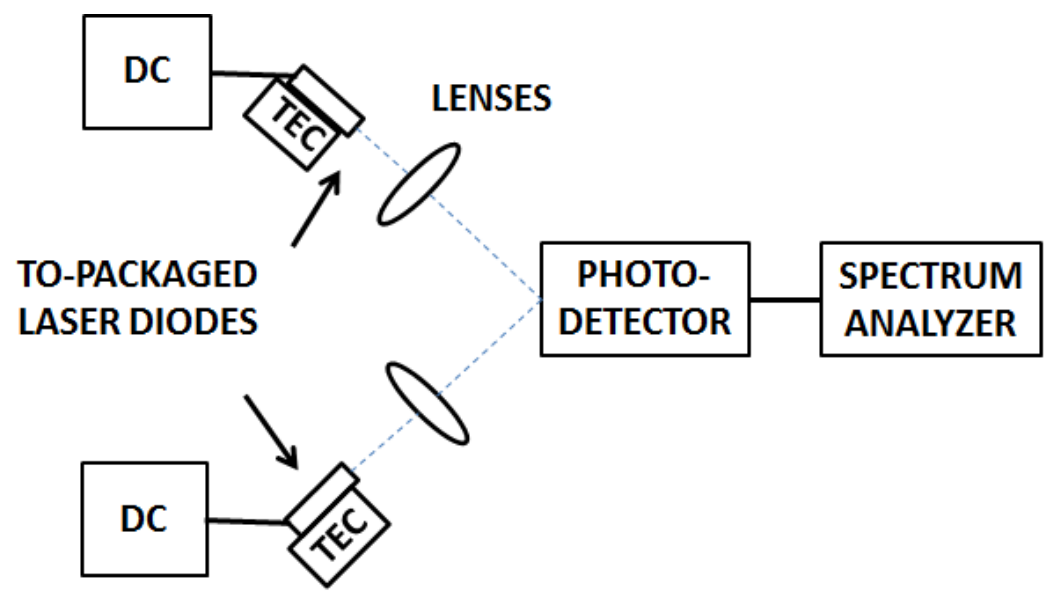

Fig.5. Beating two lasers together to get a linewidth measurement.

\section{CONCLUSIONS}

We have realised $\mathrm{InGaN} / \mathrm{GaN}$ distributed feedback laser diodes emitting at a single wavelength in the $42 \mathrm{X} \mathrm{nm}$ wavelength range. The lateral grating design offers a simplified fabrication route without regrowth and with more easily achievable feature sizes over first order gratings.

The DFB LDs demonstrated relatively high threshold current which made pulsed operation necessary. This is thought to be mainly due to the epi-material performance. Future work will focus on achieving narrow linewidth, $\mathrm{CW}$ operation via an optimised grating design and improved laser material. Single mode GaN laser devices of this type will find applications in laser cooling, medical imaging and communications. 
Acknowledgements: This work was supported by the Innovate UK project Coolblue (project number 132543), and the European Union Eurostars project, Blucom (project number 10509).

\section{REFERENCES}

[1] T.-C. Wu, Y.-C. Chi, H.-Y. Wang, C.-T. Tsai, and G.-R. Lin, "Blue Laser Diode Enables Underwater Communication at 12.4 Gbps," Sci. Rep., vol. 7, p. 40480, Jan. 2017.

[2] H. Leinen, D. Glässner, H. Metcalf, R. Wynands, D. Haubrich, and D. Meschede, "GaN blue diode lasers: a spectroscopist's view," Appl. Phys. B, vol. 70, no. 4, pp. 567-571, 2000.

[3] A. Shiner, "Development of a frequency stabilized 422-nm diode laser system and its application to a $88 \mathrm{Sr}+$ single ion optical frequency standard," no. December, 2006.

[4] S. P. Najda, P. Perlin, M. Leszczynski, T. J. Slight, W. Meredith, M. Schemmann, H. Moseley, J. A. Woods, R. Valentine, S. Kalra, P. Mossey, E. Theaker, M. MacLuskey, G. Mimnagh, and W. Mimnagh, "A multiwavelength (u.v. to visible) laser system for early detection of oral cancer," in Progress in Biomedical Optics and Imaging - Proceedings of SPIE, 2015, vol. 9328.

[5] S. Masui, K. Tsukayama, T. Yanamoto, T. Kozaki, S. I. Nagahama, and T. Mukai, "CW operation of the firstorder AlInGaN $405 \mathrm{~nm}$ distributed feedback laser diodes," Japanese J. Appl. Physics, Part 2 Lett., vol. 45, no. 46, pp. 1223-1225, 2006.

[6] D. C. Sanz, J. M. Rorison, and S. Yu, "InGaN/GaN MQW Laser Diodes with 4th Order FIB-etched Gratings," in Quantum Electronics and Laser Science Conference, 2005, p. JTuC82.

[7] H. Schweizer, H. Gräbeldinger, V. Dumitru, M. Jetter, S. Bader, G. Brüderl, A. Weimar, A. Lell, and V. Härle, "Laterally Coupled InGaN/GaN DFB Laser Diodes," Phys. status solidi, vol. 192, no. 2, pp. 301-307, 2002.

[8] L. Hou, M. Haji, I. Eddie, H. Zhu, and J. H. Marsh, "Laterally coupled dual-grating distributed feedback lasers for generating mode-beat terahertz signals," Opt. Lett., vol. 40, no. 2, p. 182, 2015.

[9] T. J. Slight, O. Odedina, W. Meredith, K. E. Docherty, and A. E. Kelly, "InGaN/GaN Distributed Feedback Laser Diodes With Deeply Etched Sidewall Gratings," IEEE Photonics Technol. Lett., vol. 28, no. 24, pp. 28862888, Dec. 2016.

[10] R. Phelan, W. H. Guo, Q. Lu, D. Byrne, B. Roycroft, P. Lambkin, B. Corbett, F. Smyth, L. P. Barry, B. Kelly, J. O'Gorman, and J. F. Donegan, "A novel two-section tunable discrete mode Fabry-Pérot laser exhibiting nanosecond wavelength switching," IEEE J. Quantum Electron., vol. 44, no. 4, pp. 331-337, 2008.

[11] S. Ogita, Y. Kotaki, K. Kihara, M. Matsuda, H. Ishikawa, and H. Imai, "Dependence of spectral linewidth on cavity length and coupling coefficient in DFB laser," Electron. Lett., vol. 24, no. 10, pp. 613-614, 1988.

[12] Y. Wang, Y. Yang, S. Zhang, L. Wang, and J. J. He, "Narrow Linewidth Single-Mode Slotted Fabry Perot Laser Using Deep Etched Trenches," IEEE Photonics Technol. Lett., vol. 24, no. 14, pp. 1233-1235, Jul. 2012.

[13] T. J. Slight, A. Yadav, O. Odedina, W. Meredith, K. E. Docherty, E. Rafailov, and A. E. Kelly, "InGaN/GaN Laser Diodes with High Order Notched Gratings,” IEEE Photonics Technology Letters, 2017. 